\title{
Le dépistage de la vulnérabilité psychique des personnes atteintes de cancer : un débat
}

\section{Screening for psychological vulnerability in cancer patients: a debate}

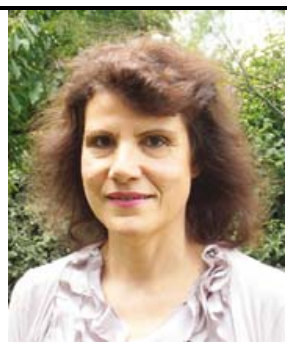

\author{
M.-F. Bacqué \\ (C) Springer-Verlag France 2013
}

Le quinzième congrès mondial de l'IPOS (International Psycho-Oncology Society) nous a offert à Rotterdam les derniers échos des grandes innovations psychosociales proposées aux patients atteints de cancer et à leurs professionnels. Vous trouverez ici quelques synthèses des sessions auxquelles j'ai pu assister.

\section{L'expression émotionnelle frustrée augmente la détresse}

Les patients qui partagent peu avec leur partenaire, bien qu'ils éprouvent d'importantes émotions et pensées, ressentent plus de symptômes dépressifs. De même que le niveau de détresse s'élève chez ceux qui ne reçoivent que peu d'écoute et de compréhension alors qu'ils ont de hauts besoins.

Les personnes déprimées avant leur cancer ont une survie moins longue (cancer du sein).

Le risque de suicide augmente chez les patients atteints de cancer (nous le savons hélas depuis longtemps).

\section{Le besoin d'information diagnostique varie en fonction du niveau d'éducation}

Ellen Smets et ses collègues de l'université d'Amsterdam ont proposé une revue de question très complète des demandes de communication, par les patients, de leur diagnostic de cancer en fonction de leur niveau social. Toutes les études consultées étaient empiriques, mais portaient sur des échantillons représentatifs.

Les auteures tentaient de répondre à l'interrogation «But do all patients want to know everything ?»

\section{M.-F. Bacqué $(\square)$}

Rédactrice en chef de Psycho-Oncologie

Université de Strasbourg, France

e-mail : mfbacque@club-internet.fr
- Les hommes plus âgés, qui sont anxieux et peu éduqués ne souhaitent que peu d'information ;

- les patients célibataires et peu éduqués préfèrent avoir peu de détails sur leur cancer ;

- les personnes faiblement diplômées réduisent leur participation à la décision et recherchent moins de seconde opinion sur leur diagnostic ;

- les personnes insécures quant à leur compréhension, celles qui sont plus anxieuses préfèrent moins d'information. De même que celles qui disent vouloir rester optimistes ou qui affirment avoir une confiance élevée en leur médecin, toutes rechercheront moins d'information sur le diagnostic et laisseront le médecin décider.

Conclusion, il est important d'adapter au maximum cette information, de la concevoir "sur mesure " pour chaque patient (le terme «tayloring », « tailler sur mesure » l'information est particulièrement juste dans ce cas...). Je traduis encore leur dernière phrase : «À la question : le patient 2013 est-il informé, décisionnaire, autoritaire et critique ? Nous répondrons que l'image d'un patient rationnel, non équivoque serait au mieux une abstraction, mais le plus souvent une caricature $»$.

\section{La modification des comportements cancérogènes après le cancer est décevante (aux États-Unis)}

Les barrières au changement de comportements à risque sont la temporalité, le fait de ne pas être sûr de ce qui est important, le fait de ne pas être prêt à changer (Suzanne Dalton).

Pour Kevin Stein, si $65 \%$ des cancers sont liés aux styles de vie, deux tiers des morts par cancer découlent de ces comportements.

Mais ceux qui ont eu un cancer changent-ils leurs habitudes pour des styles de vie plus sains?

La réponse est non. La majorité arrête bien de fumer (mais seulement un peu plus de $50 \%$ ); enfin, $5 \%$ suivent un régime et ont une activité physique (nous sommes aux États-Unis). 
Le groupe des patients atteints de cancer était cependant déjà plus sédentaire que la population générale. La plupart des « survivants » sont cependant hautement motivés pour changer.

Selon Anja Mehnert de Leipzig, $64 \%$ des survivants retournent au travail ou continuent à travailler. Si la majorité est motivée pour le travail, on comprend aisément que plus le niveau socio-économique est faible, moins le retour au travail est effectif.

Jane Maker du Macmillan Cancer Support Service a mis au point, avec son équipe, un Survivorship Program (programme de survie) disponible pour les deux millions de personnes qui survivent avec un cancer au Royaume-Uni. Leur « Recovery Package » (pack de réhabilitation) comprend des ressources électroniques (adaptables sur tout smartphone), des résumés des traitements auprès des médecins généralistes et une surveillance nationale de tous les patients. À ce propos, Alex Mitchell nous a fait tenir en main l'application pour smartphone intitulée «Cancer Stories » permettant d'écouter à tout moment de la journée des personnes qui ont eu un cancer et le racontent sur de petites vidéos. Il est possible d'interagir avec elles et d'autres par l'intermédiaire d'un "Peer Support Online » (soutien en ligne par un pair), ce qui donnera des idées aux associations de patients de plus en plus dynamiques en France. De plus, cela permet aux jeunes formés à la vidéo ou au graphisme de créer des applications performantes pour limiter l'isolement des malades.

L'exercice physique est plus que jamais considéré comme le « Cancer superdrug » (le supermédicament du cancer), les résultats se maintiennent avant, pendant et après le cancer.

J'ai beaucoup apprécié la courbe du changement de comportements après un cancer : cela ressemblait au Squiggle Game de Winnicott ; une série d'allers-retours formaient une courbe pour le moins emberlificotée... Changer d'habitudes après un cancer reste pour le moins ambigu...

\section{Les effets du dépistage : pour ou contre ?}

Le débat fait rage. Certains ne voient pas d'effet du dépistage sur la communication de leurs problèmes par les patients, d'autres comme Alex Mitchell montrent que l'acceptabilité du screening est d'environ $40 \%$ par les patients (étude portant sur 1200 d'entre eux), tandis que $43 \%$ des cliniciens la jugent utile. Pour en effet reprendre l'expérience de la Dutch Society for Psycho-Oncology sur 970 patients, ses chercheurs ont calculé que 17 heures ont été nécessaires pour recruter en moyenne un patient. Ils ont pu identifier que $38 \%$ avaient un haut niveau de détresse tandis que $46 \%$ réclamaient un soutien psychosocial. Leur travail de dépistage leur a semblé insatisfaisant et trop coûteux en temps dans un service déjà surchargé et pour une capacité de générer des ressources psychosociales insuffisantes.
Alex Mitchell, psychiatre à Londres, soutient au contraire que le dépistage augmente la communication auprès du malade. Malheureusement, les offres de soutien psychosocial sont souvent insuffisantes dans les hôpitaux. James Coyne qui enseigne à Rutgers (New Jersey, États-Unis) et aux Pays-Bas, pense, de son côté que le dépistage de la détresse pourrait démontrer l'intérêt d'embaucher plus de professionnels adaptés et d'augmenter les services rendus. Cependant, plutôt que d'utiliser le temps des professionnels à effectuer un dépistage de routine, il vaudrait mieux les employer à faire directement du bien aux malades. Pour lui, le dépistage n'a pas fait la preuve de son intérêt. Le dépistage rationalise en effet les services offerts aux patients plutôt que de leur donner l'opportunité de discuter de leurs problèmes ("Unless care is taken in implementing touchscreen screening for distress, interacting with a touchscreen can become just another barrier to patients having ready face-to-face contact with peer and professional resources »). Le screening empêche l'accès au soutien des patients qui le demandent alors même qu'ils ne sont pas dépistés comme en ayant besoin, du coup, il aggrave les inégalités sociales entre ceux qui y ont accès et les autres non (One of the unintended consequences of introduction of screening is that it could involve rationing of services and restricting of services to many patients who are currently accessing them). Finalement, le screening peut conduire à un remplissage routinier de questionnaires qui seront simplement placés dans le dossier papier ou électronique du patient sans action supplémentaire («Without adequate resources, the process of screening can degenerate into patients completing a screening instrument that is simply lost or placed in a paper folder or electronic record without further action »).

Le débat, à l'américaine (chaque interlocuteur debout derrière un pupitre), a soulevé la salle qui n'a pas voulu s'arrêter à l'heure dite. Il y avait clairement les partisans des deux bords. C'est pourquoi vous pourrez lire tous les arguments de Jim Coyne, tandis que je n'ai pas pu bénéficier du texte d'Alex Mitchell, mais les avis en faveur du dépistage seront retrouvés dans les autres contributions. Soulignons que le dépistage n'est pas forcément quantitatif, il peut être réalisé par un clinicien expérimenté qui, tout en restant à la disposition et à l'écoute d'un patient, peut toutefois, par le biais de quelques questions, réaliser un diagnostic. Il en va de la subtilité du clinicien ici même. Imaginons qu'une psychopathologie soit repérée, le clinicien pourra, si la demande du patient est celle d'un soutien ou d'une psychothérapie, faire appel à un autre spécialiste afin de préciser le diagnostic, tandis qu'il poursuivra, libéré de la contrainte prescriptrice, l'accompagnement de son patient. Cela exige évidemment des « ressources psychosociales » dans les services, comme le souligne justement Jim Coyne. À défaut, ce sont évidemment les investigations diagnostiques qui seront favorisées... 
Remerciements aux experts qui ont accepté de relire des articles proposés à Psycho-oncologie et de suggérer des améliorations : Emmanuel Babin - Anne Brédart - Véronique Christophe - Silla Consoli - Geneviève Coudin - Lionel Dany - Martine Derzelle Sylvie Dolbeault - Catherine Durdux - Livia Edery - Cécile Flahault - Pierre Gagnon - Paul Greenman - Florence Joly - Cédric
Lemogne - Yves Libert - Joëlle Ligghezzollo - Frédéric Limosin Eva Louvet - Jean-Luc Machavoine - Eliane Marx - Carole Mathelin - Dominique Merg - Isabelle Merckaert - Jean-François Morère - Nicole Pélicier - Isabelle Piollet - Alice Polomeni - Sylvie Pucheu - Etienne Seigneur - Frédéric Stiefel - Claude de Tychey.

\section{Springer Link}

\section{link.springer.com}

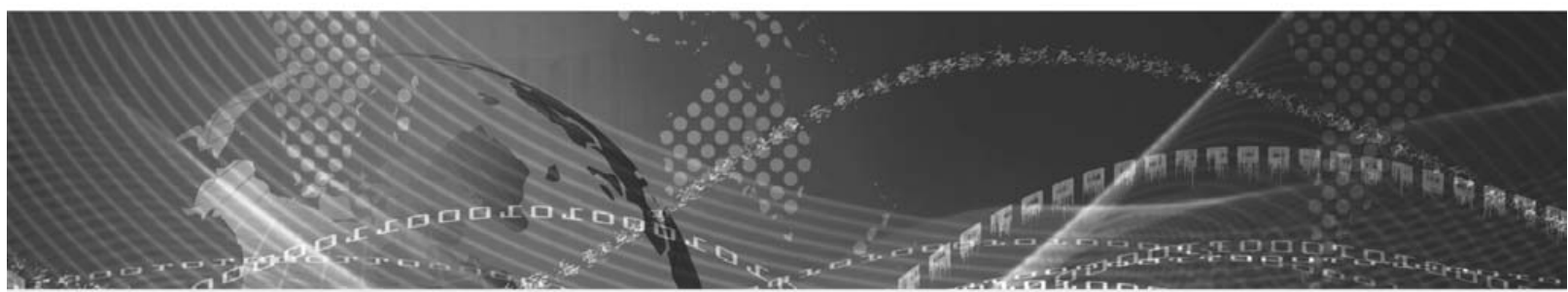

\section{SpringerLink}

Knowledge Matters. Choose SpringerLink.

- The World's Leading Scholarship

- In the Most Complete Online Collection of STM Content

- Delivered on the Fastest, Most Intelligent Research Platform We've Ever Developed

- All from Springer - A Global Leader in Scientific Publishing 